\title{
Association of a Functional Polymorphism in the Matrix Metalloproteinase-12 Promoter Region with Systemic Sclerosis in an Italian Population
}

\author{
MIRKO MANETTI, LIDIA IBBA-MANNESCHI, CINZIA FATINI, SERENA GUIDUCCI, GIOVANNA CUOMO, \\ CLAUDIA BONINO, LAURA BAZZICHI, VASILIKI LIAKOULI, ROBERTO GIACOMELLI, ROSANNA ABBATE, \\ STEFANO BOMBARDIERI, CARLOMAURIZIO MONTECUCCO, GABRIELE VALENTINI, \\ and MARCO MATUCCI-CERINIC
}

\begin{abstract}
Objective. To investigate the possible implication of the matrix metalloproteinase-12 (MMP-12) gene in the genetic predisposition to systemic sclerosis (SSc) susceptibility and clinical phenotype. Methods. The MMP-12 rs2276109 A/G functional polymorphism was selected as a genetic marker and genotyped by polymerase chain reaction-restriction fragment length polymorphism assay in 513 unrelated subjects of Italian white ancestry: 250 patients with SSc [146 limited cutaneous SSc (lcSSc), 104 diffuse cutaneous SSc (dcSSc)] and 263 healthy individuals.

Results. A significant difference was observed in MMP-12 rs2276109 genotype distribution between patients with SSc and controls $(\mathrm{p}=0.0003)$, and between $\mathrm{lcSSc}$ and dcSSc $(\mathrm{p}=0.003)$. The A allele frequency was significantly higher in patients with SSc than in controls $(\mathrm{p}=0.0002)$, and higher in $\mathrm{dcSSc}$ than in $\mathrm{lcSSc}(\mathrm{p}=0.003)$. After adjustment for age and sex, the homozygosity for the A allele significantly influenced the predisposition to SSc and to dcSSc (OR 2.44, 95\% CI 1.61-3.71, p < 0.0001; OR 4.69, 95\% CI 2.36-9.33, p < 0.0001, respectively). A trend toward an association between the AA genotype and lcSSc was observed $(\mathrm{p}=0.06)$. The homozygosity for the A allele was also significantly and independently associated with antitopoisomerase I antibody positivity (OR 6.39, 95\% CI 2.18-18.76, $\mathrm{p}=0.001$ ) and interstitial lung disease (OR 2.94, 95\% CI 1.25-6.95, $\mathrm{p}=0.01$ ).

Conclusion. The MMP-12 rs2276109 gene polymorphism may contribute to susceptibility to SSc, and in particular to dcSSc and pulmonary fibrosis. (First Release July 1 2010; J Rheumatol 2010;37:1852-7; doi:10.3899/jrheum.100237)
\end{abstract}

Key Indexing Terms:

SYSTEMIC SCLEROSIS GENE POLYMORPHISM

\section{SCLERODERMA}

Systemic sclerosis (SSc, scleroderma) is a life-threatening connective tissue disease characterized by autoimmunity, widespread microvascular involvement, and progressive fibrosis of the skin and a variety of internal organs including the lung, heart, kidney, and gastrointestinal tract ${ }^{1}$. SSc is thought to be a complex trait, where disease evolution is influenced by a combination of common gene variations and the effect of a wide range of environmental variables ${ }^{1,2}$.

\section{MATRIX METALLOPROTEINASE-12 DISEASE SUSCEPTIBILITY}

Epidemiological data suggest roles for different genetic factors, and association of single-nucleotide polymorphisms (SNP) with disease susceptibility or clinical characteristics has been reported ${ }^{2,3}$.

Tissue fibrosis significantly contributes to the morbidity of patients with SSc, and pulmonary fibrosis, which manifests clinically as interstitial lung disease (ILD), is a major cause of death ${ }^{4}$. The accumulation of collagen and other
From the Department of Anatomy, Histology and Forensic Medicine; Department of Biomedicine, Division of Rheumatology, AOUC, and Excellence Centre for Research, Transfer and High Education DENOthe; Department of Medical and Surgical Critical Care, Thrombosis Centre, University of Florence, Florence; Division of Rheumatology, II University of Naples, Naples; Division of Rheumatology, IRCCS Policlinico San Matteo, University of Pavia, Pavia; Division of Rheumatology, University of Pisa, Pisa; and Department of Internal Medicine and Public Health, Division of Rheumatology, University of L'Aquila, L'Aquila, Italy.

Supported by grants from the Ministero dell'Istruzione, dell'Università e della Ricerca (MIUR), the University of Florence (ex60\% to L.

Ibba-Manneschi and M. Matucci-Cerinic), and the Associazione per lo studio della Sclerosi Sistemica e delle Malattie Fibrosanti (ASSMaF onlus).

M. Manetti, PhD, Department of Anatomy, Histology and Forensic Medicine, Department of Biomedicine, Division of Rheumatology, AOUC, and Excellence Centre for Research, Transfer and High Education DENOthe; L. Ibba-Manneschi, MD, Department of Anatomy, Histology and Forensic Medicine; C. Fatini, MD, PhD; R. Abbate, MD, Department of Medical and Surgical Critical Care, Thrombosis Centre; S. Guiducci, MD; M. Matucci-Cerinic, MD, PhD, Department of Biomedicine, Division of Rheumatology, AOUC, and Excellence Centre for Research, Transfer and High Education DENOthe, University of Florence; G. Cuomo, MD; G. Valentini, MD, Division of Rheumatology, II University of Naples; C. Bonino, MD; C. Montecucco, MD, Division of Rheumatology, IRCCS Policlinico San Matteo, University of Pavia; L. Bazzichi, MD; S. Bombardieri, MD, Division of Rheumatology, University of Pisa; V. Liakouli, MD; R. Giacomelli, MD, PhD, Department of Internal Medicine and Public Health, Division of Rheumatology, University of L'Aquila.

Address correspondence to Dr. M. Manetti, Department of Anatomy, Histology and Forensic Medicine, University of Florence, Viale G.B. Morgagni 85, I-50134, Florence, Italy.E-mail: mirkomanetti@yahoo.it Accepted for publication April 1, 2010. 
extracellular matrix (ECM) components in SSc is caused by an increased ECM production by activated fibroblasts that differentiate into myofibroblasts and acquire the ability to contract $^{1,5}$. The net amount of ECM deposited by fibroblasts is regulated by a complex network of cell-matrix interactions that comprise synthetic and degradative processes ${ }^{6}$. ECM turnover is controlled by various matrix metalloproteinases (MMP) and their tissue inhibitors, which are produced by granulocytes, macrophages, endothelial cells, and myofibroblasts, and have been implicated in aberrant fibrotic tissue remodeling ${ }^{7}$. Further, MMP are key regulators of cell migration, and are involved in the control of growth factor, cytokine, and chemokine activity ${ }^{7}$. However, the role of MMP in the pathogenesis of SSc is poorly understood.

Increasing evidence indicates that MMP-12 (also known as macrophage metalloelastase) is involved in types of pathological lung tissue remodeling such as chronic pulmonary inflammation and fibrosis $8,9,10$. We have recently shown that dermal fibroblasts and microvascular endothelial cells isolated from patients with diffuse cutaneous SSc $(\mathrm{dcSSc})$ constitutively overexpress and release MMP-12 ${ }^{11}$. Interestingly, MMP-12 overproduction by SSc cells has been shown to be a permanent alteration over multiple generations in vitro ${ }^{11}$.

The human MMP-12 gene, on chromosome 11q22.3, contains a common $\mathrm{A} / \mathrm{G}$ functional $\mathrm{SNP}$ in the promoter region (rs2276109, $M M P-12-82 \mathrm{~A} / \mathrm{G}$ ) that modulates transcriptional activity in an allele-specific manner ${ }^{12}$. The A allele has a greater affinity to the activator protein-1 (AP-1) transcription factors, resulting in increased promoter activity and enhanced MMP-12 expression ${ }^{12}$.

Our aim was to verify whether the MMP-12 gene could be a potential candidate gene for SSc and to investigate its possible implication in the genetic predisposition to $\mathrm{SSc}$ susceptibility and clinical phenotype.

\section{MATERIALS AND METHODS}

Study subjects. Two hundred fifty consecutive patients with SSc, attending the outpatient clinics of the divisions of rheumatology of the universities of Florence, Pavia, Pisa, L'Aquila, and II University of Naples, were enrolled in the study. As screened on the basis of their clinical history, clinical features, and laboratory tests, patients with overlap autoimmune and/or connective tissue diseases were excluded from the study. Because of the recent demonstration that the $M M P-12$ rs2276109 variant influences lung function in adult smokers ${ }^{13}$, smoking subjects were also excluded. Two hundred sixty-three healthy unrelated nonsmoking individuals, comparable with patients for age and sex and residing in the same geographical regions and cities, were recruited from volunteer blood donors. Exclusion criteria for controls were history of autoimmune, cardiovascular, pulmonary, or any other systemic disease. All study subjects were of Italian European white ancestry, defined as all 4 grandparents being Italian white. Patients with SSc and healthy controls were representative of the whole Italian population, deriving from the northern, central, and southern areas of Italy. The study was approved by the local institutional review boards, and written informed consent was obtained from all study subjects.

Clinical evaluations. At enrollment, a detailed clinical history was recorded for each patient with SSc. Limited cutaneous SSc (lcSSc) or dcSSc sub- sets were defined according to LeRoy, et $a l^{14}$. Disease duration was calculated since the first non-Raynaud's symptom of SSc. Antinuclear, anticentromere, and antitopoisomerase I antibodies were determined following standard techniques. Antinuclear antibodies were determined using indirect immunofluorescence and HEp-2 cells as antigen substrate (Antibodies Inc., Davis, CA, USA). Anticentromere antibodies were determined by their distinctive indirect immunofluorescence pattern on HEp-2 cells. Antitopoisomerase I antibodies were determined by passive immunodiffusion against calf thymus extract, using a commercial kit (Inova Diagnostics, San Diego, CA, USA). Organ involvement was assessed as described ${ }^{15,16}$. ILD was studied with pulmonary function tests [a restrictive pattern was defined as forced vital capacity and diffusing capacity for carbon monoxide (DLCO) $\leq 70 \%]$ and high-resolution computed tomography scan. In the presence of DLCO reduction alone, patients were investigated for pulmonary arterial hypertension (PAH). Patients with a systolic pulmonary artery pressure $>40 \mathrm{~mm} \mathrm{Hg}$ at echocardiography underwent right-heart catheterization to confirm $\mathrm{PAH}^{16}$.

MMP-12 rs2276109 A/G genotyping. Genomic DNA was purified from whole peripheral blood using a QIAamp DNA blood mini kit (Qiagen, Hilden, Germany). The $M M P-12$ rs 2276109 A/G polymorphism was genotyped by polymerase chain reaction (PCR)-restriction fragment length polymorphism assay introducing a $P v u I I$ restriction enzyme site with use of the forward mismatch primer 5'-GAG ATA GTC AAG GGA TGA TAT CAG C-3' (mismatched base underlined) and the reverse primer 5'-AAG AGC TCC AGA AGC AGT GG-3' as described ${ }^{17}$. PCR was performed in a total reaction volume of $25 \mu 1$ containing $2 \mu 1$ of diluted genomic DNA, using AccuPrime SuperMix II (Invitrogen, Carlsbad, CA, USA). The amplification protocol was initial denaturation at $94^{\circ} \mathrm{C}$ for $2 \mathrm{~min}, 35$ cycles of $94^{\circ} \mathrm{C}$ for $30 \mathrm{~s}$ (denaturation), $60^{\circ} \mathrm{C}$ for $30 \mathrm{~s}$ (annealing), and $68^{\circ} \mathrm{C}$ for 1 min (extension), with a final extension at $68^{\circ} \mathrm{C}$ for 5 min using a TC-512 thermocycler (Techne, Cambridge, UK). PCR products were digested by $P v u I I$ restriction endonuclease (10 units; New England BioLabs, Beverly, MA, USA) at $37^{\circ} \mathrm{C}$ for $2 \mathrm{~h}$. The resulting fragments were separated by $3 \%$ low-melting-point agarose gel electrophoresis with ethidium bromide staining and a 100 bp DNA ladder (Invitrogen), and visualized under ultraviolet transillumination. With the rs2276109 A polymorphic base, the recognition sequence 5'-CAG/CT్-3' is modified to 5'-CAG/CTㅍ-3', which is not cut by $P v u$ II restriction enzyme (Figure 1). Genotyping was performed with blinding to disease status of subjects. All samples were genotyped in the same center to avoid genotyping inconsistencies. Control samples of predetermined genotypes and negative controls (i.e., undigested PCR products, PCR amplification without DNA template) were included in each sample set, and more than $20 \%$ of all samples were randomly selected for confirmation showing $>99 \%$ accuracy.

Statistical analysis. Statistical analysis was performed using the SPSS software for Windows, version 11.5 (SPSS, Chicago, IL, USA). Categorical and quantitative variables were respectively described as numbers, percentages, and mean \pm SD. Genotype distribution and allele frequency of the MMP-12 rs2276109 polymorphism in both patients and controls were compared using chi-squared analysis. A chi-squared test was used to compare the observed numbers of each genotype with those expected for a population in Hardy-Weinberg equilibrium. The association between MMP-12 rs2276109 polymorphism and SSc or clinical subsets was assessed using univariate logistic regression analysis under a dominant, recessive, and additive genetic model of inheritance. The dominant genetic model compares the combined MMP-12 rs2276109 AA + AG genotypes with the homozygous GG genotype (baseline). The recessive genetic model compares the MMP-12 rs2276109 AA genotype with the combined rs 2276109 $\mathrm{AG}+\mathrm{GG}$ genotypes, which form the baseline group. The additive genetic model assumes that there is a linear gradient in risk between the rs2276109 AA, AG, and GG genotypes (rs2276109 GG genotype baseline). This is equivalent to a comparison of the rs 2276109 A allele with the rs $2276109 \mathrm{G}$ allele (baseline). Variables such as age and sex were included into the multivariate model to evaluate their confounding effect. The OR with $95 \% \mathrm{CI}$ was determined. $\mathrm{P}$ values $<0.05$ were considered statistically significant.

Personal non-commercial use only. The Journal of Rheumatology Copyright (C) 2010. All rights reserved. 


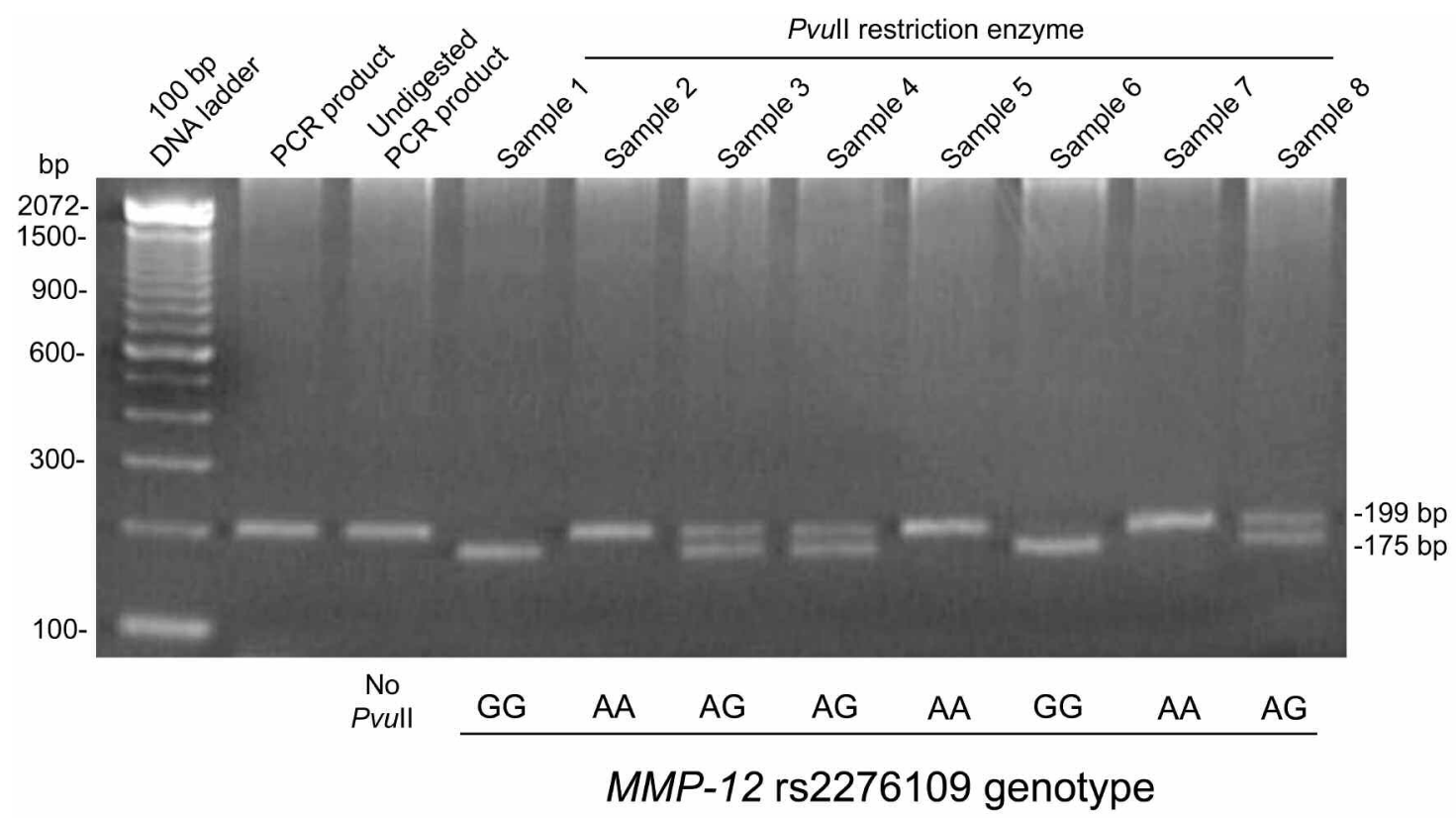

Figure 1. Analysis of the MMP-12 rs2276109 A/G gene polymorphism by PCR-restriction fragment length polymorphism assay. A $P v u I I$ restriction endonuclease site was introduced with use of a forward mismatch primer. PCR products (199 bp) were digested with $P v u I I$ restriction enzyme and subsequently separated by $3 \%$ low-melting-point agarose gel electrophoresis with ethidium bromide staining and visualized under ultraviolet transillumination. The rs2276109 A allele resulted in 1 fragment of $199 \mathrm{bp}$, while the rs $2276109 \mathrm{G}$ gene variant resulted in 2 fragments of $175 \mathrm{bp}$ and $24 \mathrm{bp}$. Results of a representative experiment are shown. Samples 1 and 6 have the GG genotype, samples 2, 5, and 7 have the AA genotype, and samples 3, 4, and 8 have the heterozygous AG genotype.

\section{RESULTS}

Demographic, clinical, and serological characteristics of the study population are reported in Table 1. No deviation from the expected population genotype proportions predicted by Hardy-Weinberg equilibrium was detected in patients with SSc or controls, at the MMP-12 rs2276109 polymorphic site.

The MMP-12 rs2276109 genotype distribution and allele frequencies found in controls were in good agreement with those previously reported in the European white population $^{12}$ (Table 2). A significant difference in MMP-12 rs2276109 genotype distribution was observed between patients with SSc and healthy individuals (chi-squared = 16.37, $\mathrm{p}=0.0003$ ), and between $\mathrm{lcSSc}$ and dcSSc subsets (chi-squared $=11.39, \mathrm{p}=0.003$; Table 2). Moreover, the rs2276109 genotype distribution was significantly different between the dcSSc and control groups (chi-squared $=24.64$, $\mathrm{p}<0.0001)$. The rs 2276109 A allele frequency was significantly higher in patients with SSc than in controls (chisquared $=13.73, p=0.0002$ ), as well as in dcSSc compared with controls (chi-squared $=19.78, p<0.0001$ ) and patients with lcSSc (chi-squared $=8.77, \mathrm{p}=0.003$; Table 2).

The MMP-12 rs2276109 A allele significantly influenced the predisposition to SSc (OR 2.26, 95\% CI 1.51-3.39, p <
Table 1. Demographic, clinical, and serological characteristics of patients with systemic sclerosis (SSc) and control subjects. Except where indicated otherwise, values are the number $(\%)$ of subjects. Disease duration was calculated since the first non-Raynaud's symptom and did not differ significantly among subsets of patients with SSc.

\begin{tabular}{lcc}
\hline Characteristics & $\begin{array}{c}\text { Patients with SSc, } \\
\mathrm{n}=250\end{array}$ & $\begin{array}{c}\text { Controls, } \\
\mathrm{n}=263\end{array}$ \\
\hline Mean age, yrs \pm SD & $55.9 \pm 12.5$ & $54.5 \pm 12.7$ \\
Sex & & \\
Male & $27(10.8)$ & $46(17.5)$ \\
Female & $223(89.2)$ & $217(82.5)$ \\
Mean disease duration, yrs \pm SD & $7.7 \pm 6.9$ & - \\
Disease subset & $146(58.4)$ & - \\
lcSSc & $104(41.6)$ & - \\
dcSSc & & - \\
Autoantibody positivity & $227(90.8)$ & - \\
ANA & $77(30.8)$ & - \\
Anti-topo I & $91(36.4)$ & - \\
ACA & & - \\
Organ involvement & $74(29.6)$ & - \\
ILD & $35(14.0)$ & \\
PAH & &
\end{tabular}

ACA: anticentromere antibodies; ANA: antinuclear antibodies; anti-topo I: antitopoisomerase I antibodies; dcSSc: diffuse cutaneous SSc; ILD: interstitial lung disease; lcSSc: limited cutaneous SSc; PAH: pulmonary arterial hypertension. 
Table 2. Genotype distribution and allele frequency of the MMP-12 rs2276109 A/G single-nucleotide polymorphism in controls, patients with SSc, and SSc clinical subsets. Values are the number (\%) of subjects with a given genotype and the frequency of the rs 2276109 A allele in patients and controls.

\begin{tabular}{lcccc}
\hline Subjects & AA & AG & GG & $\begin{array}{c}\text { A Allele } \\
\text { Frequency }\end{array}$ \\
\hline Controls, $\mathrm{n}=263$ & $171(65.0)$ & $85(32.3)$ & $7(2.7)$ & 0.81 \\
SSc, $\mathrm{n}=250$ & $202(80.8)$ & $43(17.2)$ & $5(2.0)^{*}$ & $0.89^{* *}$ \\
lcSSc, $\mathrm{n}=146$ & $108(74.0)$ & $35(24.0)$ & $3(2.0)$ & 0.86 \\
dcSSc, $\mathrm{n}=104$ & $94(90.4)$ & $8(7.7)$ & $2(1.9)^{\S}$ & $0.94^{\S \S}$ \\
Anti-topo I-positive SSc, $\mathrm{n}=77$ & $73(94.8)$ & $3(3.9)$ & $1(1.3)$ & $0.97^{\#}$ \\
Anti-topo I-negative SSc, $\mathrm{n}=173$ & $129(74.6)$ & $40(23.1)$ & $4(2.3)$ & 0.86 \\
ACA-positive SSc, $\mathrm{n}=91$ & $64(70.3)$ & $25(27.5)$ & $2(2.2)$ & 0.84 \\
ACA-negative SSc, $\mathrm{n}=159$ & $138(86.8)$ & $18(11.3)$ & $3(1.9)$ & 0.92 \\
SSc-ILD, $\mathrm{n}=74$ & $67(90.5)$ & $5(6.8)$ & $2(2.7)$ & $0.94^{\dagger}$ \\
SSc-non-ILD, $\mathrm{n}=176$ & $135(76.7)$ & $38(21.6)$ & $3(1.7)$ & 0.87 \\
SSc-PAH, $\mathrm{n}=35$ & $31(88.6)$ & $4(11.4)$ & $0(0.0)$ & $0.94^{\dagger \dagger}$ \\
SSc-non-PAH, $\mathrm{n}=215$ & $171(79.5)$ & $39(18.2)$ & $5(2.3)$ & 0.89 \\
\hline
\end{tabular}

$\mathrm{P}$ values calculated by chi-squared analysis. ${ }^{*} \mathrm{p}=0.0003$, ** $\mathrm{p}=0.0002$ for comparison with controls. ${ }^{\S} \mathrm{p}<$ 0.0001 for comparison with controls and 0.003 with $1 \mathrm{cSSc}$; $\$$ $\$<0.0001$ for comparison with controls and 0.003 with lcSSc; ${ }^{\#} \mathrm{p}<0.0001$ for comparison with controls and 0.0004 with anti-topo I-negative SSc; ${ }^{\dagger} \mathrm{p}<0.0001$ for comparison with controls and 0.03 with SSc-non-ILD; ${ }^{\dagger \dagger} \mathrm{p}=0.1$ for comparison with SSc-non-PAH. ACA: anticentromere antibodies; anti-topo I: antitopoisomerase I antibodies; dcSSc: diffuse cutaneous SSc; ILD: interstitial lung disease; lcSSc: limited cutaneous SSc; PAH: pulmonary arterial hypertension.

0.0001 ) under a recessive, but not dominant or additive genetic model of inheritance (Table 3). In particular, the $M M P-12$ rs2276109 AA genotype was strongly associated with dcSSc (OR 5.06, 95\% CI 2.51-10.18, p < 0.0001), and a trend toward association with $\mathrm{lcSSc}$ was observed (OR $1.53,95 \%$ CI $0.98-2.39, \mathrm{p}=0.06$ ). After adjustment for age and sex, the MMP-12 rs2276109 AA genotype significantly and independently remained a susceptibility factor for the disease (OR 2.44, 95\% CI 1.61-3.71, p < 0.0001), and in particular for dcSSc (OR 4.69, 95\% CI 2.36-9.33, p < 0.0001; Table 3).

The possible correlation between the MMP-12 rs2276109 polymorphism and the major serological charac- teristics and organ manifestations of SSc was also analyzed. Concerning the autoantibody status, the frequency of the MMP-12 rs2276109 A allele was significantly higher in the subset of patients carrying antitopoisomerase I antibodies in comparison to antitopoisomerase I antibody-negative SSc (chi-squared $=12.7, \mathrm{p}=0.0004)$ and controls (chi-squared $=$ $22.30, \mathrm{p}<0.0001$; Table 2). As pulmonary manifestations are considered, we observed a significant difference in MMP-12 rs2276109 genotype distribution and allele frequency between SSc-ILD and SSc-non-ILD (chi-squared = $8.16, p=0.02$; chi-squared $=4.53, p=0.03$, respectively), as well as between SSc-ILD and healthy individuals (chi-squared $=19.46, \mathrm{p}<0.0001 ;$ chi-squared $=13.93, \mathrm{p}=$

Table 3. Univariate analysis for association between the MMP-12 rs2276109 polymorphism and SSc clinical subsets according to recessive, dominant, and additive genetic models. Healthy controls were used as reference for all comparisons except anti-topo I-positive SSc versus anti-topo I-negative SSc, and SSc-ILD versus SSc-non-ILD.

\begin{tabular}{|c|c|c|c|c|c|c|}
\hline \multicolumn{7}{|l|}{ Univariate Analysis } \\
\hline $\mathrm{dcSSc}$ & $5.06(2.51-10.18)$ & $<0.0001$ & $1.28(0.28-5.92)$ & 0.7 & $1.68(0.36-7.83)$ & 0.5 \\
\hline lcSSc & $1.53(0.98-2.39)$ & 0.06 & $1.19(0.32-4.49)$ & 0.8 & $1.22(0.32-4.60)$ & 0.8 \\
\hline Anti-topo I-positive SSc & $6.22(2.15-18.03)$ & 0.0002 & $1.79(0.20-16.36)$ & 0.6 & $2.26(0.25-20.63)$ & 0.5 \\
\hline SSc & $2.44(1.61-3.71)$ & $<0.0001$ & - & - & - & - \\
\hline $\mathrm{dcSSc}$ & $4.69(2.36-9.33)$ & $<0.0001$ & - & - & - & - \\
\hline Anti-topo I-positive SSc & $6.39(2.18-18.76)$ & 0.001 & - & - & - & - \\
\hline SSc-ILD & $2.94(1.25-6.95)$ & 0.01 & - & - & - & - \\
\hline
\end{tabular}

* Adjusted for age and sex. Anti-topo I: anti-topoisomerase I antibodies; dcSSc: diffuse cutaneous SSc; ILD: interstitial lung disease; lcSSc: limited cutaneous SSc. 
0.0002; Table 2). Instead, no difference was found between SSc-PAH and SSc-non-PAH in either genotype distribution or allele frequency (chi-squared $=1.91, \mathrm{p}=0.4$; chi-squared $=2.05, \mathrm{p}=0.1$; Table 2).

The homozygosity for the MMP-12 rs2276109 A allele was significantly associated with antitopoisomerase I antibody-positivity (OR 6.22, 95\% CI 2.15-18.03, $\mathrm{p}=0.0002$ ) and ILD (OR 2.91, 95\% CI 1.24-6.83, p = 0.01), even after adjustment for age and sex (OR 6.39, 95\% CI 2.18-18.76, $\mathrm{p}=0.001$, and OR 2.94, 95\% CI 1.25-6.95, $\mathrm{p}=0.01$, respectively; Table 3). No effect of the rs2276109 A allele was observed under a dominant or additive genetic model of inheritance (Table 3).

\section{DISCUSSION}

In this case-control study, we analyzed for the first time the possible role of the MMP-12 gene in modulating both the predisposition to SSc and its clinical phenotype. In particular, we selected as a genetic marker the rs2276109 functional SNP, which is located within the $M M P-12$ gene promoter immediately adjacent to an AP-1 consensus element bound by transcription factors c-Fos and c-Jun ${ }^{12}$. The $M M P-12$ rs2276109 A allele has been shown to possess higher binding affinity to transcription factors of the AP-1 family and increased transcriptional activity in vitro ${ }^{12}$.

Our study shows that the MMP-12 rs2276109 A allele influences the susceptibility to SSc, and predominantly to the dcSSc form. Moreover, in patients with SSc the homozygosity for the MMP-12 rs2276109 A allele is significantly associated with the presence of antitopoisomerase I antibodies and ILD. Interestingly, these data are in agreement with our previous findings demonstrating that different cell types isolated from the skin of patients with dcSSc constitutively overexpress and release MMP-12 in the culture medium over multiple generations in vitro ${ }^{11}$.

SSc is a multifactorial disease in which genetic and environmental components interact in modulating its pathogenesis and evolution ${ }^{2,3}$. The search for new genetic components may shed light on SSc pathophysiology and may help to identify markers predicting major organ manifestations, such as pulmonary fibrosis or PAH, thus suggesting novel therapeutic strategies.

The MMP are a family of proteases that exert various biological effects in cell migration and ECM remodeling, as well as inflammatory and immune responses ${ }^{7,18}$. The promoters of different $M M P$ genes, including $M M P-1, M M P-3$, $M M P-9$, and $M M P-12$, contain functional SNP that have been associated with the development and progression of various diseases ${ }^{19}$. A recent case-control study found no association between SNP in the $M M P-2, M M P-9$, and MMP-14 genes and SSc in a French white population ${ }^{20}$. However, to our knowledge the possible implication of the $M M P-12$ gene in susceptibility to SSc has never been investigated. We considered $M M P-12$ as a potential candidate gene for SSc, since the MMP-12 enzyme has emerged as critical in chronic pulmonary pathologies characterized by an intense tissue remodeling, such as asthma, chronic obstructive pulmonary disease (COPD), and airway fibrosis $^{21}$. A recent study has shown that the MMP-12 rs2276109 variant is associated with the risk of COPD and correlates with lung function in children with asthma and adult smok$\mathrm{ers}^{13}$. Individuals homozygous for the A allele were at increased risk of COPD and had lower lung function compared with subjects with at least 1 copy of the G protective allele $^{13}$. This is consistent with our findings showing that patients with SSc carrying the homozygous AA genotype are at increased risk of developing ILD.

Alveolar macrophages are the principal source of MMP-12 in the lung ${ }^{21}$. MMP-12 protein levels and enzymatic activity are increased in the induced sputum of patients with COPD, and are inversely correlated with measures of lung function ${ }^{22}$. MMP-12 has been shown to act as a downstream mediator of transforming growth factor ß-induced pulmonary inflammation and fibrosis ${ }^{9}$. Mice carrying a targeted deletion of the MMP-12 gene were resistant to the induction of pulmonary fibrosis and showed decreased expression of different profibrotic genes ${ }^{10,21}$. Moreover, treatment with bleomycin induced MMP-12 messenger RNA expression in lung tissue, while the MMP inhibitor batimastat prevented bleomycin-induced pulmonary fibrosis in wildtype mice ${ }^{8}$. In addition, it has been shown that MMP-12-dependent migration of extrapulmonary myofibroblast progenitors contributes to posttransplant fibrosis in the lung 23 .

Our work suggests that the MMP-12 rs2276109 SNP contributes to the susceptibility to SSc and might be an indicator of severe skin and pulmonary fibrosis in the disease course. It consequently provides a basis for further analysis of this SNP in independent and larger case-control cohorts, as replication is needed to validate genetic associations. Further functional studies are warranted to fully elucidate the role of the MMP-12 gene in the pathogenesis of SSc. Modulation of MMP-12 expression and activity might offer new targeted therapeutic strategies to control the progression of fibrosis in SSc.

\section{REFERENCES}

1. Varga J, Abraham D. Systemic sclerosis: a prototypic multisystem fibrotic disorder. J Clin Invest 2007;117:557-67.

2. Agarwal SK, Tan FK, Arnett FC. Genetics and genomic studies in scleroderma (systemic sclerosis). Rheum Dis Clin North Am 2008;34:17-40.

3. Granel B, Bernard F, Chevillard C. Genetic susceptibility to systemic sclerosis: from clinical aspect to genetic factor analyses. Eur J Intern Med 2009;20:242-52.

4. Hesselstrand R, Scheja A, Akesson A. Mortality and causes of death in a Swedish series of systemic sclerosis patients. Ann Rheum Dis 1998;57:682-6.

5. Varga J, Bashey RI. Regulation of connective tissue synthesis in systemic sclerosis. Int Rev Immunol 1995;12:187-99.

6. Wynn TA. Common and unique mechanisms regulate fibrosis in Personal non-commercial use only. The Journal of Rheumatology Copyright @ C 2010. All rights reserved. 
various fibroproliferative diseases. J Clin Invest 2007;117:524-9.

7. Pardo A, Selman M. Matrix metalloproteases in aberrant fibrotic tissue remodeling. Proc Am Thorac Soc 2006;3:383-8.

8. Lagente V, Le Quement C, Boichot E. Macrophage metalloelastase (MMP-12) as a target for inflammatory respiratory diseases. Expert Opin Ther Targets 2009;13:287-95.

9. Kang HR, Cho SJ, Lee CG, Homer RJ, Elias JA. Transforming growth factor (TGF)-beta 1 stimulates pulmonary fibrosis and inflammation via a Bax-dependent, bid-activated pathway that involves matrix metalloproteinase-12. J Biol Chem 2007;282:7723-32.

10. Matute-Bello G, Wurfel MM, Lee JS, Park DR, Frevert CW, Madtes DK, et al. Essential role of MMP-12 in Fas-induced lung fibrosis. Am J Respir Cell Mol Biol 2007;37:210-21.

11. Serratì S, Cinelli M, Margheri F, Guiducci S, Del Rosso A, Pucci $\mathrm{M}$, et al. Systemic sclerosis fibroblasts inhibit in vitro angiogenesis by MMP-12-dependent cleavage of the endothelial cell urokinase receptor. J Pathol 2006;210:240-8.

12. Jormsjo S, Ye S, Moritz J, Walter DH, Dimmeler S, Zeiher AM, et al. Allele-specific regulation of matrix metalloproteinase-12 gene activity is associated with coronary artery luminal dimensions in diabetic patients with manifest coronary artery disease. Circ Res 2000;86:998-1003.

13. Hunninghake GM, Cho MH, Tesfaigzi Y, Soto-Quiros ME, Avila L, Lasky-Su J, et al. MMP12, lung function, and COPD in high-risk populations. N Engl J Med 2009;361:2599-608.

14. LeRoy EC, Black C, Fleischmajer R, Jablonska S, Krieg T, Medsger TA Jr, et al. Scleroderma (systemic sclerosis): classification, subsets and pathogenesis. J Rheumatol 1988;15:202-5

15. Liakouli V, Manetti M, Pacini A, Tolusso B, Fatini C, Toscano A, et al. The $-670 \mathrm{G}>\mathrm{A}$ polymorphism in the FAS gene promoter region influences the susceptibility to systemic sclerosis. Ann Rheum Dis 2009;68:584-90.

16. Manetti M, Liakouli V, Fatini C, Cipriani P, Bonino C, Vettori S, et al. Association between a stromal cell-derived factor 1

(SDF-1/CXCL12) gene polymorphism and microvascular disease in systemic sclerosis. Ann Rheum Dis 2009;68:408-11.

17. Woo M, Park K, Nam J, Kim JC. Clinical implications of matrix metalloproteinase- $1,-3,-7,-9,-12$, and plasminogen activator inhibitor-1 gene polymorphisms in colorectal cancer.

J Gastroenterol Hepatol 2007;22:1064-70.

18. Sternlicht MD, Werb Z. How matrix metalloproteinases regulate cell behavior. Annu Rev Cell Dev Biol 2001;17:463-516.

19. Ye S. Polymorphism in matrix metalloproteinase gene promoters: implication in regulation of gene expression and susceptibility of various diseases. Matrix Biol 2000;19:623-9.

20. Wipff J, Dieude P, Avouac J, Tiev K, Hachulla E, Cracowski JL, et al. Association of metalloproteinase gene polymorphisms with systemic sclerosis in the European Caucasian population. J Rheumatol 2010;37:599-602.

21. Garbacki N, Di Valentin E, Piette J, Cataldo D, Crahay C, Colige A. Matrix metalloproteinase 12 silencing: a therapeutic approach to treat pathological lung tissue remodeling? Pulm Pharmacol Ther 2009;22:267-78.

22. Demedts IK, Morel-Montero A, Lebecque S, Pacheco Y, Cataldo D, Joos GF, et al. Elevated MMP-12 protein levels in induced sputum from patients with COPD. Thorax 2006;61:196-201.

23. Sato M, Hirayama S, Lara-Guerra H, Anraku M, Waddell TK, Liu $\mathrm{M}$, et al. MMP-dependent migration of extrapulmonary myofibroblast progenitors contributing to posttransplant airway fibrosis in the lung. Am J Transplant 2009;9:1027-36. 